\title{
Preparing the Open Dialogue Approach for Implementation in the United States
}

- Recovery is a process of revival and resiliency, grounded in hope, empowerment, and a supportive network, with which a productive and meaningful life is restored and prevails. This perspective grows from the knowledge that people can and do overcome many challenges thought to be posed by mental health issues or addiction.

- Effective recovery-oriented psychosocial treatments exist and continue to be developed, and there is a great need to disseminate and implement these approaches into clinical practice.

- Open Dialogue is a recovery-oriented psychosocial approach that has been found to be effective with persons in acute psychiatric crisis. The approach emphasizes patient-centered care and engaging an individual's family and social network. Drs. Jaakko Seikkula, Birgitta Alakare, and Jukka Aaltonen developed the approach at Keropudas Hospital / University of Jyväskylä in Tornio, Finland, and it is now being used in many places in Europe.

- The Open Dialogue approach (therapy and organizational change) has been found to improve outcomes for acute psychosis, such as fewer and shorter hospitalizations, less recidivism, reduced neuroleptic medication dosage, improved likelihood of employment, and greater improvements in functioning (Aaltonen, Seikkula, \& Lehtinen, 2011; Seikkula, Aaltonen, Alakare, Haarakangas, \& Lehtinen, 2006; Seikkula, Alakare \& Aaltonen, 2011).

- As enthusiasm for the approach increases, there is a realization that there is a need for therapy / program implementation manuals and fidelity tools to help clinicians and leaders. There is a need for lessons learned in adapting to new cultures.

\section{Present UMass Global Partnership Project}

Drs. Douglas Ziedonis and Mary Olson are leading a multi-disciplinary team at the University of Massachusetts Medical School / UMass Memorial Health Care to develop implementation tools (manuals, fidelity scales, etc.) that are needed to implement and evaluate the Open Dialogue approach in the United States. Dr. Jaakko Seikkula and his team at the University of Jyväskylä in Finland are working closely with the UMass project leadership team which also includes Drs. Jon Delman, Daniel Fisher, Lisa Mistler, and others. The manuals and fidelity scales the project will develop are needed in the field and will help the implementation of the Open Dialogue approach in new settings. We already anticipate adapting the Finnish model to fit with the US culture and system by including peer specialists and recovery-oriented language and concepts.

\section{Guiding Principles of the Open Dialogue Approach:}

1. IMMEDIATE HELP

2. FAMILY / SOCIAL NETWORK PERSPECTIVE

3. FLEXIBILITY AND MOBILITY

4. RESPONSIBILITY

5. PSYCHOLOGICAL CONTINUITY

6. TOLERANCE OF UNCERTAINTY

7. DIALOGUE (\& POLYPHONY)

The Open Dialogue approach is distinguished by the integration of two key elements, the therapeutic "dialogic process" of clinical practice and the organizational change adaptations of the treatment system.

The products created in this project are an important step for future research and program implementation. The project will create:

- Psychotherapy-focused manual \& fidelity tool for clinical interactions with individuals and families

- System-focused manual \& fidelity tool for assessing program/ organizational implementation of the Open Dialogue model

\section{Value of this work:}

This project is a crucial step for clinicians, program leaders, and researchers in the United States and throughout the world to further investigate the Open Dialogue approach. These written materials will provide important groundwork for future endeavors in staff training and implementation activities needed to apply and further evaluate this recovery-oriented model in new settings.

\section{References:}

Aaltonen, J., Seikkula, J., \& Lehtinen, K. (2011). Comprehensive open-dialogue approach I: Developing a comprehensive culture of need-adapted approach in a psychiatric public health catchment area the Western Lapland Project. Psychosis, 3, 179 - 191. Seikkula, J., Aaltonen, J., Alakare, B., \& Haarakangas, K. (2006). Five-year experience of first-episode nonaffective psychosis in open-dialogue model. Psychotherapy Research 16(2): 214-228. Seikkula, J., Alakare, B., \& Aaltonen, J. (2011). The comprehensive open-dialogue approach(II). Long-term stability of acute psychosis outcomes in advanced community care: The Western Lapland Project. Psychosis, 3, 1-13.

Project Team Leaders: Douglas Ziedonis, MD, MPH (Principal Investigator); Mary Olson, PhD (Co-Investigator) Funder: Foundation for Excellence in Mental Health Care

Contact Person: Makenzie.Tonelli@umassmed.edu 